\title{
Avaliando "Depressões": dos Critérios Diagnósticos às Escalas Psicométricas
}

\author{
Makilim Nunes Baptista ${ }^{1}$ \\ Universidade São Francisco, Campinas-SP, Brasil
}

\section{RESUMO}

O termo "depressão" pode ser utilizado para se referir a uma gama bastante abrangente de diagnósticos e expressões de sintomas, de maneira que duas pessoas podem apresentar sintomas completamente diferentes uma da outra e, mesmo assim, ambas terem diagnóstico semelhante. Nesse sentido, as escalas podem ser fundamentais no processo de rastreio, auxílio no diagnóstico e intervenção de pacientes com sintomatologia clinicamente significativa de depressão. Este artigo tem como objetivo discutir duas temáticas atuais que envolvem a avaliação psicológica. A primeira se refere a diferentes apontamentos (descrição, importância, críticas) sobre os critérios dos manuais psiquiátricos referentes aos transtornos depressivos. Na segunda temática, são discutidas características das escalas que avaliam sintomatologia depressiva e suas especificidades, já que elas podem ser distintas entre si, em vários aspectos, como teorias de base, dimensionalidade, número de itens, tipo de resposta, especificidades do ciclo de vida, formas de aplicação, dentre outros.

Palavras-chave: transtornos de humor; transtornos afetivos; psicometria; escalas; avaliação psicológica.

\section{ABSTRACT - Evaluating "Depressions": from diagnostic criteria to psychometric scales}

The term "depression" can be used to refer to a fairly wide range of diagnoses and expressions of symptoms, so that two people may have completely different symptoms from each other, and yet both may have a similar diagnosis. Therefore, scales can be fundamental in the screening process, aiding in diagnosis and intervention with patients presenting clinically significant symptoms of depression. This article aims to discuss two current issues in psychological assessment. The first refers to different notes (description, importance, criticism) regarding the criteria in psychiatric manuals related to depressive disorders. The second theme discusses characteristics of the scales that evaluate depressive symptomatology and their specificities are discussed, since they may differ from each other, in several aspects such as basic theories, dimensionality, number of items, response type, life cycle specificities, application forms, among others.

Keywords: mood disorders; affective disorders; psychometry; scales; psychological evaluation.

RESUMEN - Evaluación de las "depresiones": desde los criterios diagnósticos hasta las escalas psicométricas

El término "depresión" puede ser utilizado para referirse a una gama bastante amplia de diagnósticos y expresiones sintomáticas, de tal forma, que dos personas pueden presentar síntomas completamente diferentes una de la otra y, aun así, ambas pueden tener un diagnóstico similar. En este sentido, las escalas pueden ser fundamentales en el proceso de rastreo, ayuda en el diagnóstico e intervención en pacientes con sintomatología clínicamente significativa de depresión. Este artículo tiene como finalidad discutir dos temáticas de actualidad, que involucran a la evaluación psicológica. La primera se refiere a diferentes apuntes (descripción, importancia, críticas) sobre los criterios de los manuales psiquiátricos referentes a los trastornos depresivos. En la segunda temática, se discuten características de las escalas que evalúan la sintomatología depresiva y sus particularidades, dado que las mismas pueden ser muy diferentes entre sí en varios aspectos, tales como teorías de base, dimensionalidad, número de ítems, tipo de respuesta, particularidades del ciclo de la vida, formas de aplicación, entre otros.

Palabras clave: trastornos del humor; trastornos afectivos; psicometría; escalas; evaluación psicológica.

O termo "depressão" frequentemente é utilizado para designar uma condição psiquiátrica, tal como o Episódio Depressivo Maior (EDM) ou o Transtorno Depressivo Maior (TDM), no entanto, atualmente vem sendo utilizado de forma difusa. A depressão pode ser considerada como um fenômeno heterogêneo em termos de variação de sinais (manifestações observáveis), sintomas (subjetivos) e síndrome (combinação de sinais e sintomas). Aparte, os sintomas típicos, por exemplo do EDM, podem ser observados nas pessoas sem diagnóstico, em variações diversas de intensidade, no cotidiano, e em outros transtornos psiquiátricos. Logo, a importância em se diferenciar quadros depressivos bem estabelecidos pelos manuais psiquiátricos (DSM e CID) dos sintomas depressivos, que podem estar presentes em diversas situações da vida (American Psychiatric Association, 
2014; Monroe \& Anderson, 2015; Organização Mundial da Saúde, 1993). Neste artigo, o termo "depressão" será utilizado como sinônimo de EDM e/ou TDM e, sintomatologia depressiva, como um conjunto de descritores que podem estar presentes, em algum momento, na vida, mas que não necessariamente descreve um diagnóstico completo categórico.

O número crescente de diagnósticos de transtornos depressivos vem sendo considerado como um problema de saúde pública mundial de grandes proporções. A depressão atinge basicamente todo o ciclo vital, gerando consequências importantes em várias áreas, desde o absenteísmo escolar/laboral, custos para os sistemas de saúde, sofrimento psicológico, implicações nos relacionamentos sociais, até o aumento da probabilidade de morbi-mortalidade, na associação com suicídio (Baptista, 2004; Santos, Marcon, Espinosa, Baptista, \& Paulo, 2017; World Health Organization, 2004).

A prevalência de depressão (EDM) durante a vida é de 16,2\% e, em amostras representativas dos Estados Unidos, 6,6\% durante o último ano. Além de um episódio de depressão durar, em média, quatro meses, geralmente vem acompanhado de outro transtorno comórbido. Mais de 330 milhões de pessoas sofrem de depressão no mundo, ou seja, cerca de $4,4 \%$ de toda a população, e somente $20 \%$ recebe tratamento adequado quando diagnosticado de forma correta (Kessler et al., 2003). Um estudo meta-analítico envolvendo 90 pesquisas de 30 países de diversos continentes, desde 1994 a 2014, com mais de um milhão de pessoas avaliadas, apontou 12,9\% de prevalência ponto; $7,2 \%$ no último ano e $10,8 \%$ na vida, tendo a América do Sul a maior prevalência agregada. No entanto, é importante citar que essa inflação na prevalência ponto se deu porque, em vários estudos, foram utilizadas escalas, e não entrevistas estruturadas diagnósticas (Lim et al., 2018), lembrando que as entrevistas estruturadas são ferramentas próprias para o diagnóstico e, as escalas geralmente são utilizadas no apoio das decisões diagnósticas e/ou como parâmetro de melhora, como linha de base para melhora de intervenções medicamentosas e/ou psicoterápicas (Baptista, 2010).

No Brasil, a prevalência é de 5,8\% da população, sendo apenas menor do que a dos Estados Unidos quando a comparação é realizada nas Américas, superando todos os países vizinhos da América do Sul (Kessler, Chiu, Demler, \& Walters, 2005; Kessler et al., 2003; WHO, 2017). Ainda no Brasil, aproximadamente 11,2 milhões de pessoas têm depressão, com maior prevalência em mulheres (10,9\% x 3,9\%), na área urbana e nas regiões sul e sudeste, das quais $7,6 \%$ relatam que foram diagnosticadas por um profissional de saúde com esse transtorno (Instituto Brasileiro de Geografia e Estatística - IBGE, 2013).

Os transtornos depressivos são fenômenos altamente complexos marcados pela presença de componentes biológicos (ex. genéticos, saúde), psicológicos (personalidade) e/ou culturais sociais (suporte familiar e social, preconceito), e diferentes sintomas podem estar associados a características clínicas diversas (Fried et al., 2011; Fried \& Nesse, 2015; Lux \& Kendler, 2010). Provavelmente, pela complexidade da conjunção de todas as variáveis contidas nessas três grandes áreas, e pelas possibilidades de apresentação de combinação dos sintomas, que pode ser diferente em cada indivíduo; os tratamentos medicamentosos também parecem não ter os efeitos esperados e, muitas vezes, não muito diferentes do que os placebos, o que também pode ser generalizado para tratamentos psicoterápicos, especificamente no contexto nacional (Baptista, 2010; Fried $\&$ Nesse, 2015).

Os sintomas depressivos podem aparecer uma única vez e ser caracterizado como um episódio ou podem ser reincidentes. Os sintomas também podem ser desencadeados em diferentes quantidades, intensidades (ex. moderado, severo) e dimensionalidades (agrupamentos diversos) no decorrer do transtorno, além de poderem ser avaliados enquanto traço e/ou estado (Fried et al., 2016). Ainda, alguns sintomas específicos podem ser indicadores de novas reincidências ou de melhora nos tratamentos (Bringmann, Lemmens, Huibers, Borsboom, \& Tuerlinckx, 2014).

Os fatores de risco podem estar intimamente ligados ao desencadeamento e remissão de episódios depressivos e seus subtipos. Por exemplo, pontos de inflexão podem ser gerados por uma situação de trabalho cronicamente estressante e/ou desprazerosa, reduzindo a resiliência e precipitando insônia ou outros sintomas, ou uma notícia ruim provinda de um telefonema familiar pode ser suficiente para propiciar uma cadeia de reações na estabilidade humoral de uma pessoa já instável, gerando sintomas depressivos com intensidade, frequência e duração necessários para um diagnóstico (van de Leemput et al., 2014).

Vários pesquisadores vêm debatendo sobre a importância e existência do diagnóstico de subtipos de expressão de sintomatologia, no intuito de se ter informações mais específicas do cliente no caso de intervenções mais promissoras, mesmo porque cinco décadas de pesquisa nessa área ainda não são suficientes para demarcar todos os subtipos possíveis (Pae, Tharwani, Marks, Masand, \& Patkar, 2009; van Loo, Jonge, Romeijn, Kessler, \& Schoevers, 2012). Alguns subtipos na sintomatologia dos transtornos depressivos possuem características diferenciadas, com a presença de determinados sintomas em detrimento de outros, fatores de risco e disparadores distintos, além de proposições de intervenções diferentes.

Os subtipos podem ser divididos em categorias, como: baseados nos sintomas (ex. melancólica, psicótica); de base aetiológica (ex. orgânica, induzida por drogas, proveniente de traumas); baseada no início dos sintomas (ex. início tardio, sazonal); de gênero (ex. em mulheres); e de tratamento resistente. Alguns dos subtipos 
encontrados na literatura são: depressão com ansiedade, aguda, melancólica, depressão adulta após trauma infantil, reativa a estresse de separação, pós-parto, no final do ciclo vital, psicótica, atípica, bipolar, secundária à utilização de drogas ou condições médicas, com sintomas somáticos, com ataques de pânico, traços obsessivos, acompanhada de doenças físicas e, com características de pseudodemências (Goldberg, 2011; Harald \& Gordon, 2012; Lamers et al., 2010; Lichtenberg \& Belmaker, 2010; Piccinelli \& Wilkinson, 2000). A título de exemplo, a depressão melancólica pode ter como característica específica a perda de peso/diminuição de apetite, insônia no final do ciclo do sono, falta de responsividade a estímulos e piora dos sintomas na parte inicial do dia. Combinações específicas de sintomatologia depressiva presentes nos subtipos de depressão podem indicar que as intervenções medicamentosas e/ou psicoterápicas devem levar em consideração tais especificidades no diagnóstico, a fim de se aumentar a probabilidade de eficácia nos tratamentos. Para tanto, o diagnóstico baseado em critérios bem definidos é fundamental.

\section{Alguns Apontamentos aos Critérios dos Manuais Psiquiátricos}

Os manuais psiquiátricos vigentes podem ser considerados como organizações taxionômicas dos diagnósticos de transtornos mentais e, especificamente relatado por este manuscrito, nos transtornos depressivos, de humor e/ou afetivos (APA, 2014; OMS, 1993). Como exemplo, o Diagnostic and Statistical Manual of Mental Disorders, DSM-5 (APA, 2014), sugere o diagnóstico de depressão quando presentes cinco de um total de nove sintomas, pelo menos estando presentes um ou dois dos principais sintomas, ou seja, o humor deprimido e a anedonia. Os demais sintomas são referentes a ganho ou perda de peso, insônia ou hipersonia, agitação ou retardo psicomotor, fadiga/perda de energia, sentimentos de inutilidade/culpa, baixa concentração e ideação suicida.

Apesar de várias críticas aos critérios diagnósticos, é importante citar que os manuais são critérios universalizados sobre psicopatologias, que possibilitam padronizações no diagnóstico e intervenções, além de facilitar a comunicação entre profissionais de saúde (Borsboom, 2008; Widiger \& Samuel, 2005). O diagnóstico de depressão vem sendo utilizado como uma importante ferramenta na Psiquiatria/Psicologia e deve vir acompanhado da compreensão dos fatores de risco, protetivos e demais variáveis associadas à patologia, já que elas serão fundamentais no processo de busca de informações para intervenções mais eficazes (Hawton, Comabella, Haw, \& Saunders, 2013; Kendler, Zachar, \& Craver, 2011).

Interessante notar que essa forma de classificação permite que um indivíduo possua sintomas completamente diferentes de outro também diagnosticado como tendo um transtorno depressivo, o que favorece a possibilidade de haver 227 perfis diferentes de sintomas, principalmente porque vários dos sintomas podem ocorrer com ausência ou presença da condição e/ou variação da frequência, intensidade e duração da condição, tais como insônia ou hipersonia, retardo ou agitação psicomotora, perda ou ganho de peso. O número de perfis pode aumentar exponencialmente se utilizadas todas as possibilidades dos sintomas, como insônia primária, intermediária e secundária, ou ainda se forem utilizadas, ao invés de combinações binárias (ter ou não ter o sintoma), possibilidades de respostas tipo Likert, elevando os perfis sintomatológicos a mais de 14000 , principalmente quando avaliados por escalas. Ainda, dois indivíduos com a mesma quantidade de sintomas listados por um manual psiquiátrico e/ou pontuação em uma escala de depressão podem ter incapacidades completamente diferentes (Fried \& Nesse, 2015; Zimmerman, Ellison, Young, Chelminski, \& Dalrymple, 2015).

Outro complicador no perfil clínico, além das várias possibilidades de combinação dos sintomas da depressão, diz respeito aos próprios sintomas de depressão, que também podem ser encontrados em outros transtornos, devendo o clínico estar atento ao diagnóstico diferencial, como o Transtorno de Ansiedade Generalizada apresenta sintomas como fadiga, dificuldade de concentração, irritabilidade (sintoma comum em crianças e adolescentes com depressão), perturbação do sono, dentre outros, sintomas que podem ser similares aos experimentados por alguém diagnosticado com depressão (APA, 2014; Beard et al., 2016; Borsboom \& Cramer, 2013).

A questão do diagnóstico, bem como da intervenção, ainda perpassa pelos paradigmas em avaliação psicológica, mais especificamente as visões de depressão enquanto categórica (existência ou não do diagnóstico), dimensional (em que parte do contínuo entre felicidade $\mathrm{x}$ tristeza ou normalidade $\mathrm{x}$ anormalidade o indivíduo se encontra) ou mesmo nas análises de redes (causas e efeitos entre os sintomas do próprio transtorno). Independentemente do paradigma do fenômeno (modelo formativo, reflexivo, etc.) (para mais detalhes ver Borsboom, 2008; Schimittmann et al., 2013; Widiger \& Samuel, 2005), o processo de avaliação é muito maior do que simplesmente o uso de testagem, e o clínico pode utilizar diferentes e múltiplos métodos no diagnóstico e diagnóstico diferencial.

Métodos de avaliação, como entrevistas estruturadas, escalas psicométricas, métodos projetivos, anamneses, diários, observação, consultas aos prontuários passados e atuais, entrevistas com familiares e pessoas próximas, dentre outros, podem ser utilizados na complementação de informações fundamentais para uma compreensão mais global dos casos clínicos e, consequentemente, para uma intervenção mais adequada. Até mesmo métodos mais modernos de acompanhamento de mudança de sintomas com a avaliação de micro níveis, a partir de dispositivos eletrônicos (celulares, tablets, etc.) podem ser importantes no entendimento do relacionamento entre 
situações diárias e causa e efeito de sintomas, o que refletiria na própria intervenção (Baptista, Hauck-Filho, \& Borges, 2017; Wichers, 2014).

Essas informações coletadas na utilização de multimétodos podem ser fundamentais para instrumentalizar o clínico na utilização de intervenções individualizadas específicas, além do que a avaliação dependerá de uma série de fatores contextuais, que devem ser respondidos a partir de pontos fulcrais, como o objetivo da avaliação, o tempo disponível para tal, os métodos que o clínico possui "expertise", o contexto (clínica particular, ambulatórios, enfermarias), dentre outros (para maiores esclarecimentos sobre o processo avaliativo, ver Baptista \& Borges, 2017).

Os critérios expressos nos manuais psiquiátricos são seguidos por grande parte dos pesquisadores e clínicos em saúde mental, apesar de críticas e outras propostas que são naturais a quaisquer tipos de modelos diagnósticos (Kotov et al., 2017). Os critérios diagnósticos para a depressão sofreram algumas modificações no decorrer das últimas décadas, no entanto, no caso de alguns transtornos existente no DSM-5 (APA, 2014) são basicamente os mesmos há mais de 40 anos, com poucas modificações nos sintomas principais, também chamados de "core symptons".

Como historiado por Lux e Kendler (2010), os critérios diagnósticos de depressão foram criados por clínicos experientes, no início da década de 1970 (Feighner, Robins, Woodruff, Winokur, \& Munoz, 1972), influenciado por alguns estudos da década de 1950 (Cassidy, Flanagan, Spellman, \& Cohen, 1957), sobre pacientes com sintomas de melancolia, maníaco-depressivos e outras condições médicas. No entanto, até a década de 2000, poucas pesquisas examinaram as características psicométricas dos sintomas do diagnóstico de Transtorno Depressivo Maior (Parker, 2005; Regier et al., 2013; Zimmerman, Chelminski, McGlinchey, \& Young, 2006a).

Apesar do diagnóstico em depressão utilizar a presença obrigatória de sintomas principais, a qualidade e a combinação destes podem ser diferentes de pessoa para pessoa, sendo que o diagnóstico categórico (ex. ter cinco dos nove critérios) parece ser restrito para garantir uma caracterização mais precisa, levando-se em consideração a heterogeneidade da combinação dos sintomas e dos perfis existentes em cada diagnóstico. Um dos grandes questionamentos atuais se refere a somatória do número de sintomas, no diagnóstico do tipo politético (ter alguns sintomas específicos e combinados), ou mesmo se a somatória de escores nas escalas, traduz um resultado real, já que se parte do princípio de que todos os sintomas teriam um mesmo peso, equivalência e/ou função na depressão, o que provavelmente não é verdadeiro (Fried, Nesse, Zivin, Guille, \& Sen, 2014; Fried \& Nesse, 2015).

Além disso, alguns sintomas presentes no diagnóstico de depressão podem ser disparados e/ou cronificados pelo próprio uso de medicamentos, o que distorceria a avaliação do fenômeno. Como apontam Bech (2006) e Fried e Nesse (2015), princípios diferentes nos medicamentos psiquiátricos podem afetar sono, peso (apetite), funções sexuais, dentre outros sintomas. Logo, a diminuição de alguns sintomas pode ser anulada pelo disparo de outros, caso o clínico leve apenas em consideração a quantidade dos sintomas presentes no diagnóstico.

A existência de um maior número de sintomas não está necessariamente associada com a piora do quadro, já que a relação entre os sintomas e sua causalidade pode ser mais importante do que a quantidade de sintomas vivenciados. Muitas vezes, a presença de apenas um sintoma aumenta a probabilidade do disparo de outros sintomas ou mesmo alguns sintomas possuem mais consequência no prejuízo psicossocial do indivíduo. Logo, eles também devem ser compreendidos em suas relações e qualidade (Faravelli, Servi, Arends, \& Strik, 1996; Fried \& Nesse, 2014). Essa questão da quantidade e qualidade dos sintomas também parece ser fundamental para as escalas que avaliam sintomas depressivos, já que, de maneira geral, não há distinção (ou peso) dos sintomas nas somatórias dos escores desses métodos.

Muitas entrevistas estruturadas trabalham com perguntas dos dois principais sintomas de depressão (humor deprimido e anedonia) e, caso o indivíduo responda negativamente a ambas, a avaliação não é positivada para um possível diagnóstico. No entanto, como afirmam Pepper e Nieuwsma (2006) e Solomon, Haaga, e Arnow (2001), em muitos casos as pessoas podem ser consideradas como apresentando sintomas nos limiares do diagnóstico ou possuem uma subsíndrome depressiva. Assim, podem passar desapercebidamente por uma avaliação pouco atenta, ou mesmo casos em que os sintomas estão começando a se manifestar, mas não possuem ainda todos os critérios para diagnóstico categórico, ou que não permanecem por, no mínimo, duas semanas como especificado nos critérios dos manuais psiquiátricos. Além disto, algumas escalas hetero ou autoaplicativas não possuem todos os sintomas referidos nos manuais psiquiátricos e outras não sofreram mudanças e atualizações no decorrer do tempo.

Diversas pesquisas vêm questionando o quanto todos os critérios (sintomas) expressos nos manuais psiquiátricos são realmente necessários para diagnóstico. Sintomas como ganho/perda de peso, indecisão, ideação suicida, agitação/retardo psicomotor, vêm sendo apontados como tendo pouca contribuição nos critérios diagnósticos (Bagby, Ryder, Schuller, \& Marshall, 2004; Zimmerman, McGlinchey, Young, \& Chelminski, 2006b, 2006c). Outros sintomas, não necessariamente presentes como critérios nos manuais psiquiátricos, vêm sendo apontados como de fundamental importância no diagnóstico, como desamparo, ruminação, desesperança, diminuição de motivação ou capacidade de iniciar comportamentos para atingir objetivos 
(diminished drive), falta de responsividade a estímulos, dentre outros (Abramson, Metalsky, \& Alloy, 1989; Baptista, 2012; Beck, Rush, Shaw, \& Emery, 1997; Fried et al., 2016; McGlinchey, Zimmerman, Young, \& Chelminski, 2006). Dessa forma, além do diagnóstico, a utilização de escalas específicas pode ser de muita relevância no processo de avaliação clínica.

\section{As Diferentes Escalas de Avaliação de Depressão}

Como abordado anteriormente, métodos variados podem ser utilizados para a avaliação de depressão. Além disto, não só escalas psicométricas podem auxiliar no diagnóstico da depressão, mas também descritores específicos dos métodos reflexivos (também denominados de projetivos) e outras escalas que podem ter sessões ou dimensões que avaliam sintomatologia depressiva. Por exemplo, no Brasil, Ely, Nunes, e Carvalho (2014) realizaram, em 2013, um levantamento no Sistema de Avaliação de Testes Psicológicos - SATEPSI, encontrando $8 \%$ de todo o material aprovado que avaliavam aspectos e/ou sintomatologia depressiva, como as escalas tradicionalmente desenvolvidas no exterior (ex. BDI) ou desenvolvidas no país (ex. EBADEP-A), bem como baterias de personalidade (ex. Bateria Fatorial de Personalidade) e métodos expressivos (ex. Rorscharch). No entanto, o presente artigo apresentará questões referentes às escalas psicométricas, até mesmo pelo espaço destinado a esse tipo de comunicação.

Para o leigo, alguns estudantes das áreas de saúde mental ou mesmo alguns profissionais com conhecimentos mais básicos na área da avaliação e/ou psicometria, as escalas de depressão podem parecer todas iguais, avaliando o mesmo fenômeno da mesma forma e gerando resultados similares. No entanto, essa concepção está equivocada. Ademais, as avaliações e diagnóstico dos transtornos depressivos podem ser influenciados pelos diversos paradigmas em psicometria, como os formativos, reflexivos, análise de redes, micro análises (Borsboom, 2008; Schmittmann et al., 2013; Wichers, 2014; Widiger \& Samuel, 2005), métodos utilizados e contextos, por exemplo, entrevistas estruturadas, escalas psicométricas, projetivos/reflexivos, objetivos da avaliação, tempo disponível (Baptista et al., 2017), dentre outras características. A possível utilização das escalas é apenas uma parte relativamente pequena, mas importante da avaliação em saúde mental (Baptista \& Borges, 2017).

É inegável que a utilização de escalas de rastreamento nos sistemas de saúde, bem como na clínica, tem diversas vantagens e pode auxiliar na prevenção de recaídas, bem como direcionar os indivíduos a tratamentos mais eficazes (Shippee et al., 2014). Nesse sentido, a United States Preventive Services Task Force (USPSTF, 2017) vem recomendando a utilização de escalas de rastreamento em pacientes de serviços de saúde primários nos Estados Unidos desde 2009, no entanto, o rastreamento pode funcionar apenas como uma primeira etapa de uma avaliação mais aprofundada, no intuito da formulação do diagnóstico e/ou diagnóstico diferencial.

$\mathrm{O}$ avaliador deve conhecer as limitações e empregos das escalas que utiliza, ter treinamento específico na avaliação e, se possível, deve se munir de métodos diferentes para a avaliação de um mesmo fenômeno, até mesmo a utilização de escalas de depressão diferentes com a finalidade de ter informações mais precisas de uma mesma sintomatologia, como ideação passiva ou ativa no suicídio, ou mesmo escalas de auto e heterorrelatos conjuntamente (Baptista et al., 2017; Bech, 2006; Frie \& Nesse, 2015; Tanner \& Haaga, 2006; Zimmerman et al., 2015).

Nem todas as escalas, até mesmo as mais utilizadas, segundo Shafer (2006), cobrem todo o contínuo do construto e/ou abrangem todas as variações de sintomas presentes nos manuais psiquiátricos. Assim, é difícil a eleição de escalas consideradas como padrão-ouro (Bagby et al., 2004; Uher et al., 2008), já que cada escala, com sua estrutura componencial, paradigma teórico, número de itens e cobertura dos diferentes componentes por itens pode ter variações diversas.

Algumas escalas são concebidas para objetivos específicos, tal como a realização de rastreamentos epidemiológicos, como a Center for Epidemiologic Studies Depression Scale - CES-D (Radloff, 1977), enquanto outras foram concebidas para uma avaliação mais pormenorizada dos sintomas no uso da clínica, como a Escala Baptista de Depressão - versão adulto - EBADEP-A (Baptista, 2012), mas também pode ser utilizada com objetivos de avaliação em maior escala. Algumas possuem menor número de itens e podem servir como triagem (screening), como versões reduzidas de escalas já conhecidas ou que foram desenvolvidas para públicos específicos, tal como a Edinburgh Postnatal Depression Scale (EPDS) (Venkatesh, Zlotnick, Triche, Ware, \& Phipps, 2014); enquanto outras foram inicialmente desenvolvidas para avaliar pacientes já diagnosticados com depressão, ou possuem itens mais facilmente endossados por estes, tal como o Beck Depression Inventory (BDI) (Beck, Sterr, \& Brow, 1996).

Ainda, há escalas que foram desenvolvidas para faixas etárias específicas, tal como o Children Depression Inventory - CDI (Kovacs, 1992), e/ou concebidas para serem aplicadas e/ou pontuadas pelo próprio entrevistador, como a Hamilton Rating Scale for Depression - HRSD (Hamilton, 1960). Algumas escalas também são construídas no sentido de evitar alguns vieses na avaliação. Por exemplo, escalas próprias para avaliação de depressão em ambientes hospitalares geralmente não contêm itens somáticos/vegetativos, muito comuns a diversas doenças físicas, o que aumentaria a pontuação de depressão, sem que esses sintomas estejam necessariamente a ela associados (Zimmerman et al., 2006a)

$\mathrm{Na}$ literatura científica, no decorrer das últimas décadas, é possível encontrar diversas escalas que foram construídas para avaliar a sintomatologia depressiva e 
auxiliar no diagnóstico da depressão, lembrando que as principais escalas de avaliação de sintomatologia depressiva conhecidas internacionalmente foram desenvolvidas por volta da década de 1960. Santor, Gregus, e Welch (2006), ao realizarem uma revisão dessas escalas, desde 1918 até a década de 2000, encontraram algo em torno de 280 medidas diferentes, bastante heterogêneas em termos de suas características, como o número de itens para avaliar sintomas, proporção de itens nos diversos domínios (cognitivo, vegetativos, afetivos, etc.), tipos de resposta (ex. dicotômico, Likert), além de concepções diferentes sobre a definição de depressão. No entanto, como os autores apontaram, apenas algumas das escalas são utilizadas mais constantemente na clínica e em pesquisas, sendo que, nem todas acompanharam mudanças, mesmo que pequenas, nos critérios diagnósticos das últimas versões dos manuais psiquiátricos, no decorrer das últimas décadas.

As escalas mais utilizadas decorrentes do estudo de Santor et al. (2006) foram o BDI, CES-D, HRSD e o Symptom Checklist SCL-90D (Derogatis, 1977). Já Baptista e Borges (2016) encontraram resultados parcialmente análogos ao estudo de Santor et al. (2006), ou seja, os principais instrumentos usados em pesquisas brasileiras, como o BDI, Hospital Anxiety and Depression Scale HADS, HRSD, CDI e EBADEP-A, a maioria adaptados para a cultura brasileira, assim como o estudo de Aros e Yoshida (2009), que encontraram o HAM-D, HADS e BDI em bases mistas (internacionais e nacionais). Ainda, escalas que avaliam sintomas de depressão podem ser parte de baterias de escalas que avaliam outros construtos ou construtos mais amplos, e que também podem ser destinados a públicos específicos, por exemplo, o infanto-juvenil (Borges, Baptista, \& Serpa, 2017).

As escalas que avaliam sintomatologia de depressão podem ser construídas de acordo com as escolhas teóricas de seus criadores e seus usos podem ser bastante específicos aos objetivos e características da avaliação (Santor et al., 2006; Snaith, 1993). Além disso, podem não somente avaliar sintomas típicos de depressão, mas também descritores que não necessariamente fazem parte dos core symptons (Santor et al., 2006). É possível encontrar escalas que avaliam somente sintomas de depressão ou que possuem dimensões e/ou descritores específicos que avaliam tal sintomatologia, tanto as de autorrelato quanto em testes expressivos (Ely et al., 2014).

Independentemente da escala utilizada, a maioria das escalas que avalia sintomatologia depressiva requer que o avaliador utilize a somatória da pontuação de todos os itens avaliados em um escore geral, que de forma padrão, será convertido em uma categoria (ex. sem sintoma, leve moderado, severo). Mais atualmente, a soma de escores das escalas de depressão para avaliar sintomatologia ou mesmo responsividade a tratamento vem sendo questionada, pois não levam em consideração a relação causal entre os sintomas ou mesmo parte-se do princípio de que todos os sintomas são iguais, apesar da somatória também ser importante do ponto de vista clínico (Fried, 2016; Fried et al., 2016). Já que muitas escalas que avaliam depressão possuem características completamente diferentes, elas podem estar avaliando, também, distintos construtos, ou pelo menos diversas interpretações de um mesmo construto. Como exemplo, pode-se citar o BDI, que avalia mais proeminentemente sintomas cognitivos $\mathrm{e}$ muitos deles não presentes nos critérios dos manuais psiquiátricos. Ainda, escalas mais curtas podem ter medidas mais válidas para avaliar mudanças em intervenções, mas, por outro lado, serem propensas a ignorar dados clínicos relevantes (Fried, 2016; Konstantinidis, Martiny, Bech, \& Kasper, 2011).

As escalas de depressão podem ter maior número de sintomas na avaliação de componentes específicos e isso deve ser considerado para seu uso adequado. Por exemplo, o BDI possui mais de $50 \%$ dos itens voltados à avaliação cognitiva, enquanto a escala de Wake não avalia esse quesito; já a EBADEP-A possui $18 \%$ dos itens que avalia a dimensão social dos sintomas depressivos, enquanto a escala de Montgomery-Asberg não avalia esse quesito (Baptista, 2012; Calil \& Pires, 1998).

Algumas escalas possuem itens com valência apenas negativa (ex. tristeza, anedonia), enquanto outras possuem também itens com semântica positiva (ex. felicidade, sono adequado), podendo avaliar o contínuo humoral entre felicidade e tristeza (Baptista, 2012; Joseph, 2006; Santor, 2006; Wood, Taylor, \& Joseph, 2010). Alguns questionamentos a respeito, por exemplo, das escalas que possuem apenas itens negativos é se o indivíduo que não pontuar nessas escalas (obter zero no escore) deve ser interpretado com alguém que não apresenta sintomas de depressão ou alguém que é "feliz/saudável" (Joseph, 2006). Assim, algumas normas de escalas podem trabalhar com esse contínuo em termos de interpretações das somatórias de pontos, indo ao encontro dos princípios da Psicologia Positiva (Baptista, 2018). De outra forma, a utilização de apenas algumas escalas nas pesquisas científicas não parece completamente salutar, já que concepções diferentes do construto, itens abordando todo o contínuo humoral (tristeza versus alegria) e suas variações (tristeza versus irritabilidade), presentes ou ausentes nos manuais, podem aumentar a riqueza de informações sobre o fenômeno. Assim, novas medidas podem ser desenvolvidas para explorar, por exemplo, a multidimensionalidade e heterogeneidade dos transtornos depressivos, ou mesmo para avaliar novos paradigmas psicométricos (Simms, 2006).

Independentemente da opção pela escala a ser utilizada, o simples fato de aplicá-la também não quer dizer que uma avaliação adequada foi realizada. Saber se o indivíduo expressa sintomatologia leve ou severa, a partir da somatória dos sintomas, também não abarca ou fornece informações completas à compreensão do caso, além do que o clínico/avaliador necessita conhecer as 
características psicométricas, modelos teóricos e outras informações importantes da escala que utiliza para auxiliar no diagnóstico e/ou acompanhar resultados de intervenção (Baptista, 2012; Faravelli et al., 1996).

Outra questão comumente encontrada na literatura diz respeito a uma mesma escala possuir evidências de diferentes configurações de dimensionalidade. Segundo Fried e Nesse (2015) e Shafer (2006), as análises fatoriais a respeito das categorias encontradas nas diversas escalas de depressão podem variar em seus resultados devido a vários fatores, como métodos estatísticos utilizados, tamanho e características das amostras, dentre outras. Estudos das principais escalas utilizadas globalmente demonstram resultados díspares, por exemplo, em termos de quantidade e compreensão dos componentes da depressão, variando entre fatores que avaliam os sintomas principais ou mais gerais, sintomas somáticos e sintomas chamados de positivos (afetos positivos) ou com semântica positiva (Shafer, 2006). Por exemplo, o BDI apresenta itens referentes a irritabilidade, pessimismo e sentimentos de estar sendo punido; o HRSD apresenta itens sobre ansiedade, sintomas genitais e hipocondria; o CES-D avalia frequência de choro, interações verbais sociais e percepção de outros como hostis (Fried et al., 2016).

Resumindo, as escalas podem ser muito diferentes entre si, construídas a partir de visões teóricas próprias, avaliando sintomas presentes ou não nos manuais psiquiátricos, possuindo número de itens diferentes em cada dimensão teórica (afetiva, cognitiva, vegetativa, social, etc,), específicas para áreas e faixas etárias diferentes (ex. hospital, idosos). Além disso, algumas escalas apresentam somente itens com valência negativa, enquanto outras possuem também itens de valência positiva. Nesse sentido, possuir conhecimentos teóricos e clínicos, bem como sobre métodos de avaliação e noções de psicometria aprofundados parece ser fundamental para auxiliar no reconhecimento da sintomatologia depressiva, bem como no diagnóstico de depressão.

\section{Considerações Finais}

A depressão não se apresenta como um fenômeno consistente, sendo condição altamente heterogênea em termos de intercâmbios dos sintomas e sem demarcações claras de limites com outras condições psicopatológicas, além do que os vários sintomas presentes nas diferentes escalas não são necessariamente equivalentes ou intercambiáveis (Fried, 2015; Monroe \& Anderson, 2015). As escalas psicométricas são ferramentas úteis na avaliação da sintomatologia depressiva e complemento do diagnóstico de depressão, sendo também a partir dos estudos com escalas que o conhecimento sobre a depressão vem se desenvolvendo. A quantidade de informações que se têm atualmente sobre transtornos depressivos está diretamente relacionada à qualidade das medidas utilizadas para avaliar o fenômeno (Santor et al., 2006).

Assim, a utilização e estudos psicométricos constantes de escalas de sintomatologia depressiva em suas diferentes configurações (teorias de base, número de itens, dimensões avaliativas) pode auxiliar na compreensão de quais sintomas são mais relevantes no diagnóstico e, em futuras modificações dos critérios dos manuais psiquiátricos, bem como auxiliar nas classificações categóricas e/ou dimensionais da depressão, refinando a forma como atualmente é avaliada a sintomatologia depressiva e auxiliando no processo de rastreamento e acompanhamento de intervenções clínicas. Como aponta Santor (2006), as diversas concepções da definição de depressão, quais os principais sintomas que avaliam esse fenômeno, como são operacionalizados nos manuais e nas escalas, e quais itens diferenciam a depressão de outros transtornos, são algumas das questões que necessitam constante investigação.

Uma compreensão mais abrangente sobre etiologia, avaliação e intervenção da depressão, modelos, paradigmas teóricos e relações clínicas está longe, mas isso é que impele a pesquisa no desvelamento de questões cruciais, já que muito conhecimento foi e está sendo desenvolvido, mas muito se tem a descobrir. Todas as áreas psicológicas, biológicas e sociais, bem como suas inter-relações, têm muito a oferecer na compreensão desse fenômeno. É fundamental que o avaliador, bem como o pesquisador e o clínico saibam o que e de que forma estão avaliando, e quais objetivos desejam alcançar com a utilização dos diversos métodos de avaliação, baseados no conhecimento científico e ético.

\section{Referências}

Abramson, L. Y., Metalsky, G. I., \& Alloy, L. B. (1989). Hopelessness depression: A theory-based subtype of depression. Psychological Review, 96(2), 358-372. doi: 10.1037/0033-295X.96.2.358

American Psychiatric Association (2014). DSM-5: Manual diagnóstico e estatístico de transtornos mentais (5a ed.). Porto Alegre: Artmed.

Aros, M. S., \& Yoshida, E. M. P. (2009). Estudos da depressão: instrumentos de avaliação e gênero. Boletim de Psicologia, 59(130), 61-76. Recuperado de http://pepsic.bvsalud.org/pdf/bolpsi/v59n130/v59n130a06.pdf

Bagby, R. M., Ryder A. G., Schuller, D. R., \& Marshall, M. B. (2004). The hamilton depression rating scale: Has the gold standard become a lead weight? American Journal of Psychiatry, 161(12), 2163-2177. doi: 10.1176/appi.ajp.161.12.2163 
Baptista, M. N. (2010). Questões sobre avaliação de processos psicoterápicos. Psicologia em Pesquisa (UFJF), 4(2), 109-117.

Baptista, M. N. (2018). Manual EBADEP-IJ - Escala Baptista de Depressão - infanto-juvenil. São Paulo: Hogrefe.

Baptista, M. N. (2012). Manual técnico da escala batista de depressão em adultos (EBADEP-A). São Paulo: Vetor.

Baptista, M. N. (2010). Questões sobre avaliação de processos psicoterápicos. Psicologia em Pesquisa, 4(2), 109-117. Recuperado de http:// pepsic.bvsalud.org/scielo.php?script=sci_arttext\&pid=S1982-12472010000200004\&lng=pt\&tlng=pt

Baptista, M. N. (2004). Suicídio e depressão: Atualizações. Rio de Janeiro: Editora Guanabara-Koogan.

Baptista, M. N., \& Borges, L. (2017). Processo de Avaliação no Contexto de Saúde. Em R. Goraybe, M. C. Miyasaki, \& M. Teodoro (Eds.), PROPSICO - Programa de Atualização em Psicologia Clínica e da Saúde (1ª ed., vol. 1, pp.41-168). Porto Alegre: Artmed Panamericana.

Baptista, M. N., \& Borges, L. (2016). Revisão integrativa de instrumentos de depressão em crianças/adolescentes e adultos na população brasileira. Avaliação Psicológica, 15(n. esp.), 19-32. Recuperado de http://pepsic.bvsalud.org/scielo.php?script=sci_arttext\&pid=S1677$04712016000300004 \& \operatorname{lng}=\mathrm{pt} \& \mathrm{t} \operatorname{lng}=\mathrm{pt}$

Baptista, M. N., Hauck-Filho, N., \& Borges, L. (2017). Avaliação em Psicologia Clínica. Em M. R. C. Lins \& J. C. Borsa, (Eds.), Avaliação Psicológica: Aspectos teóricos e práticos (pp.355-367). São Paulo: Editora Vozes.

Beard, C., Millner, A. J., Forgeard, M. J. C., Fried, E. I., Hsu, K. J., Treadway, M. T., ... Björgvinsson, T. (2016). Network analysis of depression and anxiety symptom relationships in a psychiatric sample. Psychological Medicine, 46(16), 3359-3369. doi: 10.1017/ S0033291716002300

Bech, M. D. (2006). Rating scales in depression: Limitations and pitfalls. Dialogues in Clinical Neuroscience, 8(2), 207-215. doi: 10.1.1.289.4319

Beck, A. T., Rush, A. J., Shaw, B. F., \& Emery, G. (1997). Terapia cognitiva da depressão. Porto Alegre: Artes Médicas.

Beck, A. T., Steer, R. A., \& Brown, G. K. (1996). Manual for the Beck Depression Inventory-II. San Antonio: Psychological Corporation.

Borges, L., Baptista, M. N., \& Serpa, A. L. O. (2017). Structural analysis of depression indicators scale-children and adolescents (BAID-IJ): A bifactor-ESEM approach. Temas em Psicologia, 25(2), 545-552. doi: 10.9788/TP2017.2-08

Borsboom, D. (2008). Psychometric perspectives on diagnostic systems. Journal Of Clinical Psychology, 64(9), 1089-1108. doi: 10.1002/ jclp.20503

Borsboom, D., \& Cramer A. O. J. (2013). Network analysis: An integrative approach to the structure of psychopathology. The Annual Reiew of Clinical Psychology, 9, 91-121. doi: 10.1146/annurev-clinpsy-050212-185608

Bringmann, L. F., Lemmens, L. H. J. M., Huibers, M. J. H., Borsboom, D., \& Tuerlinckx, F. (2014). Revealing the dynamic network structure of the beck depression inventory-II. Psychological Medicine, 45(4), 747-757. doi: 10.1017/S0033291714001809

Calil, H. M., \& Pires, M. L. N. (1998). Aspectos gerais das escalas de avaliação da depressão. Revista de Psiquiatria Clínica, $25(5), 240-244$. Recuperado de http://www.hcnet.usp.br/ipq/revista/vol25/n5/depre255a.htm

Cassidy, W. L., Flanagan, N. B., Spellman, M., \& Cohen, M. E. (1957). Clinical observations in manic-depressive disease: A quantitative study of one hundred manic-depressive patients and fifty medically sick controls. Journal of the American Medical Association, 164(14), 1535-1546. doi: 10.1001/jama.1957.02980140011003

Derogatis, L. R. (1977). SCL-90-R: Administration, scoring, and procedures manual for the R(evised) version - I. Baltimore: Johns Hopkins University, School of Medicine.

Ely, P., Nunes, M. F. O., \& Carvalho, L. F. (2014). Avaliação psicológica da depressão: Levantamento de testes expressivos e autorrelato no Brasil. Avaliação Psicológica, 13(3), 419-426. Recuperado de http://www.redalyc.org/html/3350/335037824014/

Faravelli, C., Servi, P., Arends, J. A., \& Strik, W. K. (1996). Number of symptoms, quantification, and qualification of depression. Comprehensive Psychiatry, 37(5), 307-315. doi: 10.1016/S0010-440X(96)90011-5

Feighner, J. P., Robins, E., Guze, S. B., Woodruff, R. A., Winokur, G., \& Munoz, R. (1972). Diagnostic criteria for use in psychiatric research. Archives of General Psychiatry, 26(1), 57-63. doi: 10.1001/archpsyc.1972.01750190059011

Fried, E. I. (2016). Are more responsive depression scales really superior depression scales? Journal of Clinical Epidemiology, 77, 4-6. doi: 10.1016/j.jclinepi.2016.05.004

Fried, E. I. (2015). Problematic assumptions have slowed down depression research: Why symptoms, not syndromes are the way forward. Frontiers in Psychology, 6(309), 1-11. doi: 10.3389/fpsyg.2015.00309

Fried, E. I., \& Nesse, R. M. (2015). Depression is not a consistent syndrome: An investigation of unique symptom patterns in the STAR ${ }^{\star} \mathrm{D}$ study. Journal of Affective Disorders, 172, 96-102. doi: 10.1016/j.jad.2014.10.010

Fried, E. I., \& Nesse, R. M. (2014). The impact of individual depressive symptoms on impairment of psychosocial functioning. PLoS ONE, 9(2), 1-7. doi: 10.1371/journal.pone.0090311

Fried, E. I., Nesse, R. M., Zivin, K., Guille, C., \& Sen, S. (2014). Depression is more than the sum score of its parts: Individual DSM symptoms have different risk factors. Psychological Medicine, 44(10), 2067-2076. doi: 10.1017/S0033291713002900

Fried, E. I., van Borkulo. C. D., Epskamp, S., Schoevers, R. A., Tuerlinckx, F., \& Borsboom, D. (2016). Measuring depression over time... Or not? Lack of unidimensionality and longitudinal measurement invariance in four common rating scales of depression. Psycholical Assessement, 28(11), 1354-1367. doi: 10.1037/pas0000275

Fried, E. I., van Borkulo, C. D., Epskamp, S., Schoevers, R. A., Tuerlinckx, F., \& Borsboom. G. (2011). The heterogeneity of "major depression". World Psychiatry, 10(3), 226-228. doi: 10.1002/j.2051-5545.2011.tb00061.x

Goldberg, D. (2011). The heterogeneity of "major depression". World Psychiatry, 10(3), 226-228. doi: 10.1002/j.2051-5545.2011.tb00061.x

Hamilton, M. (1960). A rating scale for depression. Journal of Neurology, Neurosurgery \& Psychiatry, 23(1), 56-62. doi: 10.1136/jnnp.23.1.56

Harald, B., \& Gordon, P. (2012). Meta-review of depressive subtyping models. Journal of Affective Disorders, 139(2), 126-140. doi: 10.1016/j. jad.2011.07.015

Hawton, K., Comabella, C. C., Haw, C., \& Saunders, K. (2013). Risk factors for suicide in individuals with depression: A systematic review. Journal of Affective Disorders, 147(1-3), 17-28. doi: 10.1016/j.jad.2013.01.004

Instituto Brasileiro de Geografia e Estatística [IBGE]. (2013). Pesquisa Nacional de Saúde 2013. Recuperado de ftp://ftp.ibge.gov.br/PNS/2013/ pns2013.pdf

Joseph, S. (2006). Measurement in depression: Positive psychology and the statistical bipolarity of depression and happiness. Measurement: Interdisciplinary Research and Perspectives, 4(3), 156-161.

Kendler K. S., Zachar, P., \& Craver, C. (2011). What kinds of things are psychiatric disorders? Psychological Medicine, 41(6), 1143-1150. doi: 10.1017/S0033291710001844Pub 
Kessler, R. C., Chiu, W. T., Demler, O., \& Walters, E. E. (2005). Prevalence, severity, and comorbidity of 12-month DSM-IV disorders in the National Comorbidity survey replication. Archives of General Psychiatry, 62(6), 617-627. doi: 10.1001/archpsyc.62.6.617

Kessler, R. C., Berglund, P., Demler, O., Jin, R., Koretz, D., Merikangas, K. R., ... Wang, P. S. (2003). The epidemiology of major depressive disorder results from the national comorbidity survey replication. Journal of the American Medical Association, 289(23), 3095-3105. doi: 10.1001/jama.289.23.3095

Konstantinidis, A., Martiny, K., Bech, P., \& Kasper, S. (2011). A comparison of the major depression inventory (MDI) and the beck depression inventory (BDI) in severely depressed patients. International Journal of Psychiatry in Clinical Practice, 15(1), 56-61. doi: $10.3109 / 13651501.2010 .507870$

Kotov, R., Krueger, R. F., Watson, D., Achenbach, T. M., Althoff, R. R., Bagby, R. M., ... Eaton, N. R. (2017). The hierarchical taxonomy of psychopathology (HiTOP): A dimensional alternative to traditional nosologies. Journal of Abnormal Psychology, 126(4), 454-477. doi: $10.1037 / \mathrm{abn} 0000258$

Kovacs, M. (1992). Children's depression inventory. North Tonawanda: Multi-Health Systems, Inc.

Lamers, F., Jonge, P., Nolen, W. A., Smit, J. H., Zitman, F. G., Beekman, A. T. F., \& Penninx, B. W. J. H. (2010). Identifying depressive subtypes in a large cohort study: Results from the Netherlands study of depression and anxiety (NESDA). Journal of Clinical Psychiatry, 71(12), 1582-1589. doi: 10.4088/JCP.09m05398blu

Lichtenberg, P., \& Belmaker, R. H. (2010). Subtyping major depressive disorder. Psychotherapy and Psychosomatics, 79(3), 131-135. doi: $10.1159 / 000286957$

Lim, G.Y., Tam, W.W., Lu, Y., Ho, C.S., Zhang, M.W., \& Ho, R. C. (2018). Prevalence of Depression in the Community from 30 Countries between 1994 and 2014. Scientifc Reports, 12, 8(1): 2861. doi: 10.1038/s41598-018-21243-x

Lux. V., \& Kendler, K. S. (2010). Deconstructing major depression: A validation study of the DSM-IV symptomatic criteria. Psychological Medicine, 40(10), 1679-1690. doi: 10.1017/S0033291709992157

McGlinchey, J. B., Zimmerman, M., Young, D., \& Chelminski, I. (2006). Diagnosing major depressive disorder VIII: Are some symptoms better than others? The Journal of Nervous and Mental Disease, 194(10), 785-790. doi: 10.1097/01.nmd.0000240222.75201.aa

Monroe, S. M., \& Anderson, S. F. (2015). Depression: The shroud of heterogeneity. Current Directions in Psychological Science, $24(3) 227-231$. doi: $10.1177 / 0963721414568342$

Organização Mundial de Saúde. (1993). Classificação internacional de doenças: Descrições clínicas e diretrizes diagnósticas (CID-10). Porto Alegre: Artes Médicas.

Pae, C. U., Tharwani, H., Marks, D. M., Masand, P. S., \& Patkar, A. A. (2009). Atypical depression: A comprehensive review. CNS Drugs, 23(12), 1023-2037. doi: 10.2165/11310990-000000000-00000

Parker, G. (2005). Beyond major depression. Psychological Medicine, 35(4), 467-474. doi: 0.1017/S003329170400421

Pepper, C. M., \&, Nieuwsma, J. A. (2006). Issues in the measurement of depression: Purpose, population, and interpretation. Measurement: Interdisciplinary Research and Perspectives, 4(3), 165-169.

Piccinelli, M., \& Wilkinson, G. (2000). Gender differences in depression: Critical review. The British Journal of Psychiatry, 177(6), 486-492. doi: 10.1192/bjp.177.6.486

Radloff, L. S. (1977) The CES-D scale a self-report depression scale for research in the general population. Applied Psychological Measurement, 1(3), 385-401. doi: 10.1177/014662167700100306

Regier, D. A., Narrow, W. E., Clarke, D. E., Kraemer, H. C., Kuramoto, S. J., Kuhl, E. A., \& Kupfer, D. J. (2013). DSM-5 field trials in the United States and Canada, part II: Test-retest reliability of selected categorical diagnoses. The American Journal of Psychiatry, 170(1), 5970. doi: 10.1176/appi.ajp.2012.12070999

Santor, D. (2006). New coke, rosetta stones, and functional data analysis: Recommendations for Developing and validating new measures of depression. Measurement: Interdisciplinary Research and Perspectives, 4(3), 188-197. doi: 10.1207/s15366359mea0403 3

Santor, D. A., Gregus, M., \& Welch, A. (2006). Eight decades of measurement in depression. Measurament: Interdisciplinary Research and Perspectives, 4(3), 135-155, doi: 10.1207/s15366359mea0403 1

Santos, H. G. B., Marcon, S. R., Espinosa, M. M., Baptista, M. B., \& Paulo, P. M. C. (2017). Fatores associados à presença de ideação suicida entre universitários. Revista Latino-Americana de Enfermagem, 25(2878), 1-8. doi: 10.1590/1518-8345.1592.2878

Schmittmann, V. D., Cramer, A. O. J., Waldorp, L. J., Epskamp, S., Kievit, R.A., \& Borsboom, D. (2013). Deconstructing the construct: A network perspective on psychological phenomena. New Ideas in Psychology, 31(2), 43-53. doi: 10.1016/j.newideapsych.2011.02.007

Shafer, A. B. (2006). Meta-analysis of the factor structures of four depression questionnaires: Beck, CES-D, Hamilton, and Zung. Journal of Clinical Psychology, 62(1), 126-146. doi: 10.1002/jclp.20213

Shippee, N. D., Rosen, B. H., Angstman, K. B., Fuentes, M. E., DeJesus, R. S., Bruce, S. M., \& Williams, M. D. (2014). Baseline screening tools as indicators for symptom outcomes and health services utilization in a collaborative care model for depression in primary care: A practice-based observational study. General Hospital Psychiatry, 36(6), 563-569. doi: 10.1016/j.genhosppsych.2014.06.014

Simms, L. J. (2006). The future of depression measurement research. Measurement: Interdisciplinary Research and Perspectives, 4(3), 169-174.

Snaith, P. (1993). What do depression rating scales measure? British Journal of Psychiatry, 163(3), 293-298. doi: 10.1192/bjp.163.3.293

Solomon, A., Haaga, D. A. F., \& Arnow, B. A. (2001). Is clinical depression distinct from subthreshold depressive symptoms? A review of the continuity issue in depression research. Journal of Nervous and Mental Disease, 189(8), 498-506. Recuperado de https://www. researchgate.net/profile/Dave_Haaga/publication/11815351_Is_Clinical_Depression_Distinct_from_Subthreshold_Depressive Symptoms_A_Review_of_the_Continuity_Issue_in_Depression_Research/links/573a0c3708ae9f741b2c9e4c.pdf

Tanner, M., \&, Haaga, D. A. F. (2006). Prescription Needed for the Ninth Decade of Measuring Depression. Measurement: Interdisciplinary Research and Perspectives, 4(3), 174-179.

United States Preventive Services Task Force Recommendation Statement [USPSTF]. (2017). Final Recommendation Statement Depression in Adults: Screening. Recuperado de https://www.uspreventiveservicestaskforce.org/Page/Document/RecommendationStatementFinal/ depression-in-adults-screening1

Uher R., Farmer A., Maier W., Rietschel M., Hauser J., Marusic A., ... Aitchison, K. J. (2008). Measuring depression: Comparison and integration of three scales in the GENDEP study. Psychological Medicine, 38(2), 289-300. doi: 10.1017/s0033291707001730

van de Leemput, I. A., Wichers, M., Cramer, A. O. J., Borsboom, D., Tuerlinckx, F., Kuppens, P., ... Scheffer, M. (2014). Critical slowing down as early warning for the onset and termination of depression. Proceedings of the National Academy of Sciences of the United States of America, 111(1), 87-92. doi: 10.1073/pnas.1312114110 
van Loo, A. M., Jonge, P., Romeijn, J. W., Kessler R. C., \& Schoevers, R. A. (2012). Data-driven subtypes of major depressive disorder: A systematic review. BMC Medicine, 10(156), 1-12. doi: 10.1186/1741-7015-10-156

Venkatesh, K. K., Zlotnick, C., Triche, E. W., Ware, C., \& Phipps, M. G. (2014). Accuracy of brief screening tools for identifying postpartum depression among adolescent mothers. Pediatrics, 133(1), e45-e53. doi: 10.1542/peds.2013-1628

Wichers, M. (2014). The dynamic nature of depression: A new micro-level perspective of mental disorder that meets current challenges. Psychological Medicine, 44(7), 1349-1360. doi: 10.1017/S0033291713001979

Widiger, T. A., \& Samuel, D. B. (2005). Diagnostic categories or dimensions? A question for the DSM-V. Journal of Abnormal Psychology, 114(4), 494-504. doi: 0.1037/0021-843X.114.4.494

Wood, A. M., Taylor, P. J., \& Joseph, S. (2010). Does the CES-D measure a continuum from depression to happiness? Comparing substantive and artifactual models. Psychiatry Research, 177(1-2), 120-123. doi: 10.1016/j.psychres.2010.02.003

World Health Organization [WHO]. (2017). Depression and other common mental disorders: Global health estimates. Recuperado de http://apps. who.int/iris/bitstream/10665/254610/1/WHO-MSD-MER-2017.2-eng.pdf

World Health Organization [WHO]. (2004). The global burden of disease: 2004 update. Recuperado de www.who.int/healthinfo/global_burden_ disease/GBD_report_2004update_full. pdf

Zimmerman M., Ellison, W., Young, D., Chelminski, I., \& Dalrymple, K. (2015). How many different ways do patients meet the diagnostic criteria for major depressive disorder? Comprehensive Psychiatry, 56, 29-34. doi: 10.1016/j.comppsych.2014.09.007.

Zimmerman, M., Chelminski, I., McGlinchey, J. B., \& Young, D. (2006a). Diagnosing major depressive disorder X - can the utility of the DSM-IV symptom criteria be improved? The Journal of Nervous and Mental Disease, 194(12), 893-897. doi: 10.1097/01. nmd.0000248970.50265.34

Zimmerman, M., McGlinchey, J. B., Young, D., \& Chelminski, I. (2006b). Diagnosing major depressive disorder I - A psychometric evaluation of the DSM-IV symptom criteria. The Journal of Nervous and Mental Disease, 194(3), 158-163. doi: 10.1097/01.nmd.0000202239.20315.16

Zimmerman, M., McGlinchey, J. B., Young, D., \& Chelminski, I. (2006c). Diagnosing major depressive disorder III - can some symptoms be eliminated from the diagnostic criteria? The Journal of Nervous and Mental Disease, 194(5), 313-317. doi: 10.1097/01. nmd.0000217806.16329.ff

\section{Sobre o autor}

Makilim Nunes Baptista é psicólogo, doutor pelo departamento de Psiquiatria e Psicologia Médica da Universidade Federal de São Paulo, docente do Programa de Pós-Graduação Stricto Sensu em Psicologia da Universidade São Francisco, Campinas/SP e pesquisador bolsista produtividade pelo CNPq. 